\title{
UNIVERSAL BUNDLES FOR DEFORMATIONS OF ASYMMETRIC STRUCTURES
}

BY

PAUL R. DIPPOLITO ${ }^{1}$

\begin{abstract}
Riemannian parallel transport of frames and Cartan connections can be considered as special cases of a type of structure in which the connection form on the principal bundle can transform by an arbitrary extension of the adjoint representation of the fiber group; there always exist universal deformations for asymmetric objects in any of these geometries.
\end{abstract}

The purpose of this paper is to give an elementary proof of the existence of universal objects for deformations of infinitesimally asymmetric, globally asymmetric structures in a wide range of geometries.

The class of geometries we consider is a generalization of linear connections and Cartan connections in which the connection form may transform by a more general representation of the fiber group. Within these geometries, we always require the structures under study to be both infinitesimally and globally asymmetric. Starting with a fixed geometry and a fixed principal bundle $Y \rightarrow M$ over a compact base, we construct a $Y$-bundle $\mathcal{E}^{\infty} \rightarrow \Re^{\infty}$, with a connection on each fiber, such that any deformation of asymmetric structures of the given type can be uniquely described by mapping its parameter space into $\mathfrak{\pi}^{\infty}$.

Let $H$ be a Lie group of dimension $n$ and $\theta$ a representation of $H$ on $\mathbf{R}^{m+n}$ which preserves $\{0\} \times \mathbf{R}^{n}$ and restricts so as to be isomorphic to the adjoint representation there. A $\theta$-structure on an $m$-manifold $M$ is then a principal $H$-bundle $Y \rightarrow M$ with a 1-form $\omega$ on $Y$ with values in $\mathbf{R}^{m+n}$, such that each $\omega_{y}: T_{y} Y \rightarrow \mathbf{R}^{m+n}$ is an isomorphism and $\left(R_{a}\right)^{*} \omega=\theta\left(a^{-1}\right) \omega$ for $a \in H$, and such that $\{0\} \times \mathbf{R}^{n}$ always corresponds to the vertical subspace of $T_{y} Y$. For example ( $\left.\$ 1 \mathrm{~B}\right)$, linear connections can be viewed as $\theta$-structures where $\theta$ is the sum of the standard representation of $H \subset \mathrm{GL}(m)$ on $\mathbf{R}^{m}$ and the adjoint representation on $\mathbf{R}^{n} \cong \mathfrak{h}$.

A $\theta$-structure $(Y, \omega)$ is called infinitesimally asymmetric if there is no point $y \in Y$ such that either (i) $f^{*} \omega-\left(R_{a}\right)^{*} \omega$ vanishes to infinite order at $y$ for some $a \in H$ not the identity and some local diffeomorphism $f$ fixing $y$, or (ii) there is a vector field $Z$ in $Y$ with $Z_{y} \neq 0$ such that $\varrho_{Z} \omega$ vanishes to infinite order at $y$. A different definition, given in $\S 1 \mathrm{C}$, is shown to be equivalent to this one in $\S 1 \mathrm{E}$. The $\theta$-structure is globally asymmetric if there is no nontrivial equivariant diffeomorphism $Y \rightarrow Y$ preserving $\omega$.

Received by the editors October 10, 1980.

1980 Mathematics Subject Classification. Primary 58H15, 32G07.

${ }^{1}$ Research partially supported by the National Science Foundation.

(C) 1982 American Mathematical Society 0002-9947/81/0000-1002/\$04.75 
The main result can then be precisely stated as follows:

TheOREM. Let $M$ be a compact m-manifold and $Y$ a principal $H$ bundle over $M$. Then there is a $Y$-bundle $\mathcal{E}^{\infty}(Y, \theta) \rightarrow \Re^{\infty}(Y, \theta)$ with a smooth $\theta$-structure on each fiber which is infinitesimally and globally asymmetric, and on which the connection forms and all their higher order invariants are continuous, such that any other such objects can be obtained uniquely as a pull-back from this universal one.

Our methods are quite simple. We construct an abstract jet space for fields of frames, parametrized by their local invariants. Considering the connections as framings of $T_{*} Y$, we show ( $\left.§ 2 \mathrm{D}\right)$ that infinitesimally asymmetric structures' invariants-mappings are immersions, and thus can be given normal bundles. The coordinate charts used in constructing the above bundle are then defined using the spaces of sections of these normal bundles (\$3). Finally, these charts are glued together to form the global bundle ( $\$ 4 \mathrm{~A})$, which is then shown to have the universal property $(\S 4 B)$.

1. Frame field descriptions of various types of connections. This section has two purposes. First, it combines Cartan connections, linear connections, and other geometric constructions into one natural family, described at the end of $\S 1 \mathrm{~B}$. Secondly, it illustrates the extent to which the information in any of these geometries (for instance, the principal bundle structure itself) is contained in the framing of the total space $Y$ which is provided by the connection. Writing the connection as a frame field of $N$ vectors, we see that the geometry is specified by conditions on their Lie brackets, and we describe in terms of these vector fields the hypotheses which we place on the geometry ("geometry A") whose deformations are analyzed in this paper.

A. Cartan connections. To define a Cartan connection, one must first specify a symmetric space to use as a model geometry. Suppose then that $G \supset H$ are connected finite-dimensional Lie groups, $G / H$ is a manifold of dimension $m$, and $\mathfrak{g}$, $\mathfrak{h}$ are the Lie algebras.

Definition. A Cartan connection modelled on $G / H$ is a $g$-valued 1 -form $\omega$ on an $H$ principal bundle $Y$ over a smooth $m$-manifold $M$ such that: (1) $\omega_{x}: T_{x} Y \rightarrow \mathrm{g}$ is an isomorphism of vector spaces for all $x \in Y$; (2) if $A \in \mathfrak{h}$ and $A^{*}$ is the associated invariant vector field on $Y$, then $\omega\left(A^{*}\right)=A$; (3) $\left(R_{a}\right)^{*} \omega=\operatorname{ad}\left(a^{-1}\right) \cdot \omega$ for $a \in H$, where $\left(R_{a}: Y \rightarrow Y, a \in H\right)$ defines the action of $H$.

An equivalence of these structures is a commutative diagram

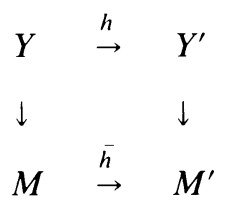

where $h, \bar{h}$ are diffeomorphisms and $h^{*}\left(\omega^{\prime}\right)=\omega$. Here the vertical arrows are the projections. 
Up to equivalence, the entire structure can be reconstructed from the pair $(Y, \omega)$. That is, the condition $h^{*}\left(\omega^{\prime}\right)=\omega$ implies that the vector fields $A^{*}$, and thus the action of $H$, are preserved by $h$. Up to equivalence we can thus replace $M$ by the orbit space of $H$ on $Y$, and we are free also to forget the $H$ action itself since it can be reconstructed from $\omega$.

Suppose now that we choose a vector space basis $e_{1}, \ldots, e_{N}$ for $\mathfrak{g}$, so that $e_{m+1}, \ldots, e_{N}$ span $\mathfrak{h} \subset \mathrm{g}$, and define vector fields $X_{1}, \ldots, X_{N}$ on $Y$ by

$$
\left\langle\omega, X_{i}\right\rangle_{x}=e_{i}, \quad i=1, \ldots, N, \quad x \in Y .
$$

These vector fields define a frame at each point of $Y$ from which we can recover $\omega$.

We have now simplified the situation substantially; our picture now consists of just a manifold with $N$ vector fields satisfying some conditions. It is reasonable to ask what the local consequences of these conditions are.

In general, the lowest order local invariants of a frame field $X_{1}, \ldots, X_{N}$ are the functions $\gamma_{j k}^{l}$ which are defined as the coefficients in

$$
\left[X_{j}, X_{k}\right]=\gamma_{j k}^{l} X_{l}
$$

(where the summation convention is used). One instance is the definition of the structure constants of $g$ by the formula

$$
\left[e_{j}, e_{k}\right]=c_{j k}^{l} e_{l} .
$$

The infinitesimal version of condition (3) of the above definition is simply

$\left(3^{\prime}\right) \gamma_{j k}^{l}=c_{j k}^{l}$ for $j>m, k$ and $l$ arbitrary.

The germs of actual connection forms, written in this notation, are the same as the germs of frame fields satisfying ( $\left.3^{\prime}\right)$. To verify this, one chooses a frame field $\left(X_{i}\right)$ in a neighborhood of $0 \in \mathbf{R}^{N}$ satisfying (3'), and notes that $H$ acts locally (in the pseudogroup sense) so that (3) holds. Using (3) and the group law, it is then possible to piece together an actual connection form on a neighborhood of $\{0\} \times H \subset \mathbf{R}^{m} \times$ $H$ whose germ (regarded as a frame field) at $(0,1)$ is isomorphic to that of $\left(X_{i}\right)$ at 0 . Note that from this point of view the geometry depends on the Lie algebras rather than the Lie groups.

Now we must discuss the possible auxiliary defining conditions for the geometries we will consider. First it is reasonable to restrict ourselves to inhomogeneous linear conditions on the $\gamma_{j k}^{l}$, and these conditions must certainly be satisfied by the model geometry, i.e. by the structure constants $c_{j k}^{l}$. Second, we note that $H$ acts on a frame field $X_{1}, \ldots, X_{N}$ by its adjoint representation on the vectorspace (namely $\mathfrak{g}$ ) generated by the $e_{i}$; this action induces transformations of the $\gamma_{j k}^{l}$ under which the conditions should be invariant.

The limitation to homogeneous linear conditions on the quantities $\gamma_{j k}^{l}-c_{j k}^{l}$ is motivated by the fact that these quantities are precisely the coefficients of the curvature

$$
\Omega=d \omega-[\omega \wedge \omega]
$$

of the Cartan connection. 
B. Linear connections and other equivariant geometries. Clearly other geometrical entities besides Cartan connections can be described in terms of framings of principal bundles. We next discuss the case of linear connections.

Given a principal $H$ bundle $Y_{0}$ of linear frames on $M(H \subset \mathrm{GL}(m))$ and a connection $\omega_{0}$ on $Y_{0}$, the horizontal subspace at each $y \in Y_{0}$ is tautologically framed since $y$ is a frame. If $Y_{0}$ is replaced by a diffeomorphic manifold $Y$ and $\omega_{0}$ by the corresponding $\tilde{\omega}$, we will need these frames on the horizontal subspaces in order to recover $\left(Y_{0}, \omega_{0}\right)$. A frame field on $Y$ is given at $y \in Y$ by these horizontal vectors $\left(X_{1}, \ldots, X_{m}\right)$ and the $X_{j}=\tilde{\omega}_{j}^{-1}\left(e_{j}\right)$ for a basis $e_{m+1}, \ldots, e_{N}$ of $\mathfrak{h}$. As in the case of Cartan connections, the manifold $M$, bundle $Y_{0} \rightarrow M$, and connection $\omega_{0}$ can then be recovered up to equivalence from $\left(Y ; X_{1}, \ldots, X_{N}\right)$. The only real difference in the two sorts of geometries lies in the conditions that the $\gamma_{j k}^{l}$ and the action of $H$ must satisfy.

The structure constants appearing in ( $\left.3^{\prime}\right)$ are only defined here if $j, k$, and $l$ are all more than $m$; the corresponding equations must be combined with $\gamma_{j k}^{l}=0$ for $j, k>m$ and $l \leqslant m$ to maintain the bundle structure on $Y$. The action of $H$ on the horizontal vectors must be that given by the inclusion $H \hookrightarrow \mathrm{GL}(m)$; this specifies the $\gamma_{j k}^{l}$ for $j>m, k \leqslant m$, and $l \leqslant m$. Finally, parallel translation must preserve the group structures on the fibers, so the $\gamma_{j k}^{l}$ must vanish for $j>m, k \leqslant m$, and $l>m$. In all, we have fixed the $\gamma_{j k}^{l}$ with $j>m$, just as in the case of Cartan connections; for notational convenience we will write $c_{j k}^{l}$ for the constants in these formulas.

These equations are again the infinitesimal form of an equation similar to condition (3) in $\$ 1 \mathrm{~A}$ which describes the behavior of a Cartan connection under translation by an element of $H$.

Just as before, we can impose auxiliary conditions on the other $\gamma_{j k}^{\prime}$ (here the torsion and curvature) to obtain other geometries, e.g. Riemannian metrics, vacuum general-relativistic space-times, and so on.

Now consider the properties held in common by the two cases of Cartan connections and linear connections.

In each the group $H$ has a representation $\theta$ given on $\mathbf{R}^{N}=\mathbf{R}^{m} \times \mathbf{R}^{N-m}$ such that

(i) $\theta$ induces a representation of $H$ on $\mathbf{R}^{m}$ by projection to the first factor, and

(ii) some vectorspace isomorphism $f:\{0\} \times \mathbf{R}^{N-m} \rightarrow \mathfrak{h}$ carries $\theta$ to the adjoint representation of $H$ on its Lie algebra $\mathfrak{h}$.

The geometry is then given by a 1-form $\omega$ on $Y$ with values in $\mathbf{R}^{N}$ such that

(iii) $\omega$ takes the tangent space of the fiber to $\{0\} \times \mathbf{R}^{N-m}$,

(iv) for each $A \in \mathfrak{h}$, the vectorfield $A^{*}$ on $Y$ given by differentiating the action of $H$ is taken to $A$ by $f \circ \omega$, and

(v) for $x \in Y$ and $a \in H$, we have $\left(R_{a}\right)^{*} \omega=\theta\left(a^{-1}\right) \cdot \omega$ where $R_{a}$ is the map giving the action of $a$ on $Y$.

The infinitesimal version of condition (v) then states that if we write vectorfields $X_{1}, \ldots, X_{N}$ on $Y$ with $\left\langle\omega, X_{j}\right\rangle \equiv e_{j}, j=1, \ldots, N$, then certain of the quantities $\gamma_{j k}^{\prime}$ in $\left[X_{j}, X_{k}\right]=\gamma_{j k}^{l} X_{l}$ must equal the corresponding structure constants $c_{j k}^{l}$ of the representation of $\mathfrak{h}$ on $\mathbf{R}^{N}$ associated to $\theta$. 
For the remainder of this paper, geometry $A$ will be any geometry defined in terms of connections of the above sort with auxiliary conditions similar to those discussed in $\$ 1 \mathrm{~A}$.

Where there are additional conditions imposed on the geometry which are not of this type, these can be simply held to one side until the construction of deformations for the less-restricted geometry is completed.

C. A remark about the kernel of $\theta$. If, in the above construction, the representation $\theta$ of $H$ on $\mathbf{R}^{N}$ has nontrivial kernel, then for any $a$ in the kernel, we have $\left(R_{a}\right)^{*} \omega=\omega: R_{a}$ is always a global symmetry of the $\theta$ structure $(Y, \omega)$. Thus we include here some remarks concerning the extension of our deformation theory to this case.

Let $1 \rightarrow H_{0} \rightarrow H \rightarrow H^{\prime} \rightarrow 1$ be an exact sequence of Lie groups, where $H_{0}$ is perhaps the kernel of $\theta$. Writing $\mathfrak{h}^{\prime}$ for the Lie algebra of $H^{\prime}$, suppose that $\theta$ preserves the fibering of $\mathbf{R}^{m} \times \mathfrak{h}\left(\cong \mathbf{R}^{N}\right)$ over $\mathbf{R}^{m} \times \mathfrak{h}^{\prime}\left(\cong \mathbf{R}^{N^{\prime}}\right)$, and that the induced action of $H$ on $\mathbf{R}^{N^{\prime}}$ factors through $H^{\prime}$. Let $\theta^{\prime}$ be the representation of $H^{\prime}$ on $\mathbf{R}^{N^{\prime}}$ so obtained. If we construct $Y^{\prime}=Y / H_{0}$ and the 1 -form $\omega^{\prime}$ which makes the diagram

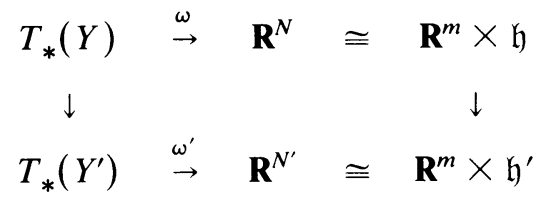

commute, we obtain an $H^{\prime}$ principal bundle $Y^{\prime} \rightarrow M$ with $\theta^{\prime}$ structure $\omega^{\prime}$.

To carry out the deformation theory of this paper in the case where $\operatorname{ker}(\theta) \neq 1$, we might begin by "reducing" the $\theta$ structure $(Y, \omega)$ to the $\theta^{\prime}$ structure $\left(Y^{\prime}, \omega^{\prime}\right)$, where $Y^{\prime}=Y / H_{0}, \omega^{\prime}$, and $\theta^{\prime}$ are as above with $H_{0}$ equal to the kernel of $\theta$. An advance is made by this simplification, since as $\theta^{\prime}$ is faithful, no $R_{a^{\prime}}: Y^{\prime} \rightarrow Y^{\prime}$ for $a^{\prime} \in H^{\prime}$ will be a symmetry of $\omega^{\prime}$. On the other hand, the functor from $(Y \rightarrow M, \omega)$ to $\left(Y^{\prime} \rightarrow M, \omega^{\prime}\right)$ is forgetful of the components of curvature lying in the Lie algebra of $H_{0}$, i.e. of the $\gamma_{j k}^{l}$ for $j, k \leqslant m$ and $l>N^{\prime}$. These extra invariants will of course be well defined on $Y^{\prime}$. Thus to extend the deformation theory to nonfaithful $\theta$, we restrict to the part of $J_{F}^{\infty}(\S 2)$ on which $\xi_{N^{\prime}+1}, \ldots, \xi_{N}$ vanish and then search for immersions of $Y^{\prime}$ rather than of $Y$ in the construction of $\S \S 2$ and 3 .

2. Invariants, jet spaces, and normal forms. In this section, we develop the basic jet space techniques in the context of abstract frame fields, and discuss the consequence of infinitesimal asymmetry. The invariants of arbitrary finite order for frame fields parametrize a jet space $J_{F}^{\infty}$; this jet space has certain structures defined on it, and there is a subspace $J_{A}^{\infty} \subset J_{F}^{\infty}$ of formal geometry $A$ structures. Infinitesimally asymmetric structures are discussed from the viewpoint of their invariant mappings, and it is seen that the latter can be easily distinguished among the mappings of $Y$ into $J_{A}^{\infty}$.

A. Normal forms for abstract frame field jets. We have seen in $\$ 1$ that Cartan connections and linear connections are essentially just fields of frames in the 
principal bundles. These fields can be written locally as ordered $N$-tuples $X_{1}, \ldots, X_{N}$ of vectorfields on (a coordinate neighborhood) $\mathbf{R}^{N}$ (in $Y$ ). We now investigate the invariants of finite order at the origin for such $N$-tuples of vectorfields under the equivalence relation given by diffeomorphisms of $\left(\mathbf{R}^{N}, 0\right)$.

These invariants are precisely the coefficients $\gamma_{j k}^{l}$ in the relations $\left[X_{j}, X_{k}\right]=\gamma_{j k}^{l} X_{l}$ evaluated at the origin and their derivatives for any multi-index $\alpha=\left(\alpha_{1}, \ldots, \alpha_{N}\right)$ given by the formula

$$
\gamma_{j k ; \alpha}^{l}=X_{1}^{\alpha_{1}} \cdots X_{N}^{\alpha_{N}} \gamma_{j k}^{l}
$$

where the ordering of the differentiations is of significance. If two such frame fields have the same invariants at the origin, then there is a diffeomorphism of $\left(\mathbf{R}^{N}, 0\right)$ taking the Taylor series of one family of vectorfields to the Taylor series of the other, as we shall see below.

To establish this claim that the above form a complete family of invariants of the Taylor series (or infinite jets) of the frame fields at the origin, we provide a normal form for any germ of frame field. This is very easy to construct. We choose new coordinates $\left(x^{1}, \ldots, x^{N}\right)$ in a neighborhood of $0 \in \mathbf{R}^{N}$ by a simple procedure. First we parametrize the integral curve of $X_{N}$ through the origin as the $x^{N}$ axis so that $X_{N}$ will equal $\partial / \partial x^{N}$ when $x^{1}=\cdots=x^{N-1}=0$. Next we use $X_{N-1}$ to similarly extend the definition of coordinates to the $\left(x^{N-1}, x^{N}\right)$ plane, so that (near the origin)

$$
X_{N-1}=\partial / \partial x^{N-1}, \quad x^{1}=\cdots=x^{N-2}=0 .
$$

By induction coordinates can be constructed on a full neighborhood of the origin so that

$$
X_{i}=\partial / \partial x^{i}, \quad x^{1}=\cdots=x^{i-1}=0, \quad \text { all } i .
$$

The coordinates are thus uniquely defined by the frame field itself, and thus the frame field is in a normal form in the new coordinates.

If the normal form expression for the frame field $\left(X_{i}\right)$ in terms of $x=\left(x^{1}, \ldots, x^{N}\right)$ is

$$
X_{j}=\partial / \partial x^{j}+a_{j}^{k}(x) \partial / \partial x^{k}, \quad 1 \leqslant j \leqslant N,
$$

then the (normal form) conditions on the $a_{j}^{k}$ are simply

$$
a_{j}^{k}(x)=0 \quad \text { when } x^{1}=\cdots=x^{j-1}=0 .
$$

Writing (for each multi-index $\alpha$ )

$$
A_{j, \alpha}^{k}:=a_{j, \alpha}^{k}(0)=(\partial / \partial x)^{\alpha} a_{j}^{k}(0),
$$

the infinitesimal form of $(*)$ is given as

$$
A_{j, \alpha}^{k}=0 \quad \text { when } \alpha_{i}=0 \text { for all } i<j .
$$

The $A_{j, \alpha}^{k}$ for which $\alpha_{i} \neq 0$ for some $i<j$ can thus be used as invariants in place of the $\gamma_{j k ; \alpha^{\prime}}^{l}$. In fact, since for any $\alpha$ the operators $X_{1}^{\alpha_{1}} \cdots X_{N}^{\alpha_{N}}$ and $(\partial / \partial x)^{\alpha}$ agree at the origin by $(*)$, we easily compute

$$
\gamma_{j k ; \alpha}^{l}=A_{j,(k)+\alpha}^{l}-A_{k,(j)+\alpha}^{l}+\sum_{\beta<\alpha}\left\{A_{k, \alpha-\beta}^{m} A_{j,(m)+\beta}^{i}-A_{j, \alpha-\beta}^{m} A_{k,(m)+\beta}^{l}\right\} ;
$$


the conditions $\left(*^{\prime}\right)$ then permit us to solve recursively for the $A_{j, \alpha}^{k}$ in terms of the $\gamma_{j k ; \alpha}^{\prime}$. Here $(j)_{i}=\delta_{i}^{j}$.

Definition. Let $\tilde{J}_{F}^{r}$ be the vectorspace consisting of germs at $0 \in \mathbf{R}^{N}$ of frame fields $X_{1}^{0}, \ldots, X_{N}^{0}$ which are in normal form with polynomial coefficients of order no greater than $(r-1)$. Let $\tilde{J}_{F}^{\infty}$ be the projective limit

$$
\tilde{J}_{F}^{\infty} \rightarrow \cdots \rightarrow \tilde{J}_{F}^{r+1} \stackrel{\pi}{\rightarrow} \tilde{J}_{F}^{r} \rightarrow \cdots \rightarrow \tilde{J}_{F}^{2},
$$

where the map $\tilde{J}_{F}^{r+1} \rightarrow \tilde{J}_{F}^{r}$ is given by deleting the $r$ th order terms in the coefficients.

To any frame field $X_{1}, \ldots, X_{N}$ on an $N$-manifold $Y$ and any $x \in Y$, the above constructions associate invariants $A_{j, \alpha}^{k}$ and thus a point of $\tilde{J}_{F}^{\infty}$ (or of $\tilde{J}_{F}^{r}$ ). The equivalence classes under the relation of mapping to the same point of $\tilde{J}_{F}^{\infty}$ (resp. of $\tilde{J}_{F}^{r}$ ) are called infinite jets (resp. $r$-jets) of abstract frame fields and form a space $J_{F}^{\infty}$ (resp. $J_{F}^{r}$ ) called the infinite jet space (resp. the $r$-jet space) of abstract frame fields.

B. Jets for geometry $A$. Let "geometry $A$ " be one of the geometries described in $\S 1$. The conditions defining the geometry (and other conditions which can be formally derived from these) will be used to define for each $r$ a subspace $J_{A}^{r}$ of $J_{F}^{r}$ describing candidates for jets of frame fields that describe objects in geometry $A$.

For each condition $\varepsilon_{l}^{j k} \gamma_{j k}^{l}=c$ in the specifications of geometry $A$ there are conditions on the frame field jets of the form

$$
\varepsilon_{l}^{j k}\left(A_{j,(k)}^{l}-A_{k,(j)}^{l}\right)=c,
$$

where $(j)$ is the multi-index with $(j)_{i}=\delta_{i}^{j}$, and the derived conditions

$$
\varepsilon_{l}^{j k}\left[A_{j,(k)+\alpha}^{l}-A_{k,(j)+\alpha}^{l}\right]=\varepsilon_{l}^{j k}\left[\sum_{\beta<\alpha}\left\{A_{k, \alpha-\beta}^{m} A_{j,(m)+\beta}^{l}-A_{j, \alpha-\beta}^{m} A_{k,(m)+\beta}^{l}\right\}\right] .
$$

These conditions will be called frame field jet conditions for the geometry $A$.

Definirion. The infinite jets (resp. $r$-jets) of abstract frame fields satisfying the frame field jet conditions for geometry $A$ will be called the infinite jets (resp. $r$-jets) of abstract connections and form a subspace of $J_{F}^{\infty}$ (resp. of $J_{F}^{r}$ ) which will be denoted $J_{A}^{\infty}\left(\right.$ resp. $\left.J_{A}^{r}\right)$.

From the representation of $H$ on $\mathbf{R}^{N}$, which is called $\theta$ in $\$ 1 \mathrm{~B}$, we obtain consistent actions of $H$ on all the $J_{F}^{r}$ by replacing $X_{i}$ by $\left(\theta\left(a^{-1}\right)\right)_{i}^{j} X_{j}$ in the equations which define the invariants. These actions restrict to actions of $H$ on the $J_{A}^{r}$ for $r=2,3, \ldots, \infty$.

We should now stress that the above definition is quite formal, and does not mention existence of an actual connection with the given jet. It would perhaps be better to have the actual spaces of $r$-jets of smooth or analytic connections. However, we have no need to compute these spaces for general geometries in order to make our constructions. The spaces

$$
J_{A, 2}^{r}=\left\{j \in J_{F}^{r} \text { : the 2-jet of } j \text { is in } J_{A}^{2}\right\}
$$

will do as well in the constructions of $\S 3$, since any actual frame field $r$-jet invariant mapping with image in $J_{A, 2}^{r}$ will have image in $J_{A}^{r}$. 
C. The canonical vectorfields on the jet space. Infinitesimal asymmetry. There is a structure on the jet space $J_{A}^{\infty}$ analogous to that structure on the space of infinite jets of maps $\mathbf{R}_{x} \rightarrow \mathbf{R}_{y}$ which is given by the Pfaffian forms $d(y)-\left(y^{\prime}\right) d(x), d\left(y^{\prime}\right)-$ $\left(y^{\prime \prime}\right) d(x), \ldots$, or as a better analogy, by the "vectorfield" $\partial / \partial x+\left(y^{\prime}\right)(\partial / \partial y)+$ $\left(y^{\prime \prime}\right)\left(\partial / \partial\left(y^{\prime}\right)\right)+\ldots$ which generates the nullspace of this infinite Pfaffian system. The structure on $J_{A}^{\infty}$ is the restriction of a similar one on $J_{F}^{\infty}$ which we will examine first.

For $j^{r} \in J_{F}^{r}$, let $\left(X_{1}^{0}, \ldots, X_{N}^{0}\right)$ be the corresponding polynomial frame field in $\tilde{J}_{F}^{r}$ defined in a neighborhood $U$ of 0 in $\mathbf{R}^{N}$. There is a smooth map $i_{0}: U \rightarrow J_{F}^{r-1}$ giving the invariants of $\left(X_{k}^{0}\right)$ of order up to $(r-1)$ at points of $U$. Define vectors $\xi_{1}^{r-1}, \ldots, \xi_{N}^{r-1}$ in the tangent space to $J_{F}^{r-1}$ at $i_{0}(0)$ by

$$
\xi_{k}^{r-1}=\left.i_{0 *}\left(\partial / \partial x^{k}\right)\right|_{x=0} .
$$

The coordinates of these vectors are invariants of $\left(X_{k}^{0}\right)$ at 0 of order no more than $r$, so the same vectors would be obtained in this way from any representative of $j^{r}$; it is also evident that the vectors depend smoothly on $j^{r}$.

If we write $j \in J_{F}^{\infty}$ as a projective $\operatorname{limit} j=\lim \left(j^{r}\right)$, we have for each $r<\infty$ and $k=1, \ldots, N$ the vectors $\xi_{k}^{r} \in T_{j^{r}}\left(J_{F}^{r}\right) \cong J_{F}^{r}$ and thus the projective limit vector

$$
\xi_{k}=\lim _{\leftarrow}\left(\xi_{k}^{r}\right) \in T_{j}\left(J_{F}^{\infty}\right) \text {. }
$$

We may thus consider the $\xi_{k}$ as "smooth" vectorfields defined on $J_{F}^{\infty}$.

If $i: Y \rightarrow J_{F}^{\infty}$ is the natural mapping giving the invariants of a frame field $X_{1}, \ldots, X_{N}$ on $Y$, then $i_{*}\left(X_{k}\right)=\xi_{k}$ for $k=1, \ldots, N$.

The higher-order conditions among the frame field jet conditions for the geometry are exactly what is needed to assure that the $\xi_{k}$ will be tangent to $J_{A}^{\infty}$; thus we can restrict the $\xi_{k}$ to obtain a similar structure on $J_{A}^{\infty}$.

DEFINITION. An infinitesimally asymmetric jet in $J_{A}^{\infty}$ is a jet which (a) is a point of trivial isotropy for the action of $H$ on $J_{A}^{\infty}$ and (b) is not a point at which the vectors $\xi_{k}$ are linearly dependent. An infinitesimally asymmetric connection in the geometry $A$ is one for which the image of the invariant mapping contains only infinitesimally asymmetric jets.

D. Immersions into $J_{F}^{r}$. We wish to simplify the recognition of invariant mappings of infinitesimally asymmetric frame fields by some construction based on the vectors $\xi_{k}$. A point $x \in J_{F}^{r}$ determines the vectors $\xi_{k}^{r-1}$ at $\pi(x) \in J_{F}^{r-1}$, and thus also their linear dependent or independence, but at one stage of the discussion we will need two derivatives, so we introduce the following open set.

Definition. For $r \geqslant 4$, let $W^{r} \subset J_{F}^{r}$ be the set of points $x \in J_{F}^{r}$ such that

(1) the vectors $\xi_{k}^{r-2}$ at $\pi^{2}(x) \in J_{F}^{r-2}$ determined by $\pi(x) \in J_{F}^{r-1}$ are linearly independent, and

(2) the isotropy of $H$ at $\pi(x) \in J_{F}^{r-1}$ is trivial.

On $W^{r}$ there is a Pfaffian system $\psi_{1}, \ldots, \psi_{K}$ of $\operatorname{rank}\left(\operatorname{dim}\left(J_{F}^{r-1}\right)-N\right)$ : the null space $\left\{\psi_{i}=0\right\}$ at $x \in W^{r}$ consists of all vectors $v \in T_{x}\left(J_{F}^{r}\right)$ with $\pi_{*}(v)$ a linear combination of the vectors $\xi_{k}^{r-1}$ at $\pi(x)$ determined by the point $x$. 
LEMMA. (1) For some neighborhood of any given point in an infinitesimally asymmetric frame field in geometry $A$, there is $r<\infty$ for which the $r$-jet invariant mapping has image in $W^{r}$.

(2) Given a smooth map $f$ of an $N$-manifold $U$ into $W^{r}, f$ is the invariant mapping of a frame field if and only if $\pi \circ f$ is an immersion and $f$ satisfies the Pfaffian system in the sense that $\left\langle\psi_{i}, f_{*} X\right\rangle=0$ for all $X \in T_{*} U$ and $i=1, \ldots, K$.

Proof. Statement (1) is trivial, as is the "only if" part of (2). Thus let $f$ satisfy the Pfaffian system and let $\pi \circ f$ be an immersion. By dimension-counting, $D(\pi \circ f)(u)$ must have image spanned by the $\xi_{k}^{r-1}$ for each $u \in U$. There is then certainly a frame field $X_{1}, \ldots, X_{N}$ on $U$ with $(\pi \circ f)_{*} X_{k}=\xi_{k}^{r-1}$; we must check that $f$ is in fact the $r$-jet invariant mapping for this frame field. Applying the identities which define the vectorfields $\xi_{k}$, we see that it suffices to check only the $\gamma_{j k}^{l}$ at each point of $U$. This is no more difficult: we know the second derivatives $I_{; j k}^{s}$ and $I_{; k j}^{s}$ of $N$ quantities $I^{s}$ (invariants of order $\leqslant r-2$ ) for which the $I_{; 1}^{s}$ form an invertible matrix.

E. The geometrical characterization of infinitesimal asymmetry.

Proposition. A frame field $\left(X_{1}, \ldots, X_{N}\right)$ on $\mathbf{R}^{N}$ is infinitesimally asymmetric at 0 if and only if there is no nonvanishing smooth vectorfield $Z$ on $\mathbf{R}^{N}$ with the Lie derivatives $\varrho_{Z}\left(X_{i}\right)$ all vanishing to infinite order at 0 . For fields in geometry A, infinitesimal asymmetry at 0 is equivalent to this condition plus the nonexistence of a diffeomorphism $h:\left(\mathbf{R}^{N}, 0\right) \rightarrow\left(\mathbf{R}^{N}, 0\right)$ for which $D h(0)=\left(h_{i}^{j}\right)$ is a transformation in the representation $\theta$ of $H$ and $h_{i}^{j} X_{j}-h_{*}\left(X_{i}\right)$ vanishes to infinite order at 0 for all $i$.

Proof. The second part of the statement is clear given the first, since in the case of infinitesimal symmetry arising from $H$ isotropy the diffeomorphism $H$ can be constructed as the transformation from normal form coordinates for $\left(X_{i}\right)$ to those for $\left(h_{i}^{j} X_{j}\right)$.

Now we introduce an auxiliary variable $t$ and lift the $X_{i}, i=1, \ldots, N$, "horizontally" to vectorfields $\tilde{X}_{i}=X_{i}+0(\partial / \partial t)$ in $\mathbf{R}_{x}^{N} \times \mathbf{R}_{t}$. Suppose $Z$ is a vectorfield on $\mathbf{R}_{x}^{N}$ as in the proposition, and introduce $\tilde{X}_{N+1}=Z+(\partial / \partial t)$ on $\mathbf{R}_{x}^{N} \times \mathbf{R}_{t}$ to complete the $(N+1)$-frame field $\left(\tilde{X}_{1}, \ldots, \tilde{X}_{N+1}\right)$; note that the $\left[\tilde{X}_{i}, \tilde{X}_{N+1}\right]$ vanish to infinite order at $(0,0)$. Putting this field in normal form using a diffeomorphism $h$ of $\left(\mathbf{R}^{N} \times \mathbf{R}, 0\right)$, we note that $h$ preserves the $t$ variable and that for each value of $t$, a frame field in normal form is induced on $\mathbf{R}^{N} \times\{t\}$ by restricting the $h_{*}\left(\tilde{X}_{i}\right)$. The invariants of these induced frames are equal to those of $\left(X_{i}\right)$ along the flow line of $Z$ through the origin. The $t$ derivatives of these invariants at 0 are invariants of $\left(\tilde{X}_{1}, \ldots, \tilde{X}_{N+1}\right)$ at $(0,0)$ which involve $t$, and thus vanish by the Lie derivative condition. Thus $\left(X_{1}, \ldots, X_{N}\right)$ is infinitesimally symmetric.

The converse is similar; we suppose that $Z_{0}$ is a vector which is annihilated by the invariant mapping of $\left(X_{1}, \ldots, X_{N}\right)$ at the origin and write $\tilde{X}_{N+1}^{0}$ for the constant vectorfield $Z_{0}+\partial / \partial t$ on $\mathbf{R}^{N+1}$. To construct the vectorfield $Z$, we put $\left(\tilde{X}_{1}, \ldots, \tilde{X}_{N}, \tilde{X}_{N+1}^{0}\right)$ in normal form at the origin by a diffeomorphism $h:\left(\mathbf{R}^{N+1}, 0\right) \rightarrow$ $\left(\mathbf{R}^{N+1}, 0\right)$ and set

$$
Z_{x}=\pi_{*}\left(h^{-1}\right)_{*}\left\{\left.(\partial / \partial t)\right|_{(x, 0)}\right\}
$$


where $\pi(x, t)=x$ for $(x, t) \in \mathbf{R}^{N} \times \mathbf{R}$. After writing $\tilde{X}_{N+1}=Z+\partial / \partial t$ and transforming to normal form for $\left(\tilde{X}_{1}, \ldots, \tilde{X}_{N+1}\right)$, it is easy to compute the required brackets $\left[Z, X_{i}\right]$ and their derivatives.

3. Deformations of an infinitesimally asymmetric, globally asymmetric geometry $A$ structure on a compact manifold. The purpose of this section is to provide the constructions which are summarized in the following theorem. Here the concrete objects $(Y \rightarrow M, \omega)$ in geometry $A$ are first given the topology of $\bigodot^{\infty}$ convergence of connection forms on compact subsets of $Y$.

THEOREM 1. Given an infinitesimally asymmetric, globally asymmetric object $(Y \rightarrow$ $M, \omega)$ in gemoetry $A$, with $M$ compact, there are

(1) a finite-dimensional vector bundle $E \rightarrow M$,

(2) a system of differential equations on $E$, and

(3) a subset $E_{A} \subset E$

such that those solutions of the system (2) which are in a $\bigodot^{\infty}$ neighborhood of the zero section and have values in $E_{A}$ are in one-to-one correspondence with the equivalence classes of those objects in geometry $A$ which are $e^{\infty}$ perturbations of the given one.

Choosing $(Y \rightarrow M, \omega)$ as in the theorem, we begin by writing the structure as a family of $N$ vectorfields $X_{1}, \ldots, X_{N}$ on $Y$. There is then as in $\S 2$ A a mapping $i_{\omega}$ : $Y \rightarrow J_{F}^{\infty}$ describing all the finite-order local invariants of this $N$-frame field on $Y$ : this map has image in the subset $J_{A}^{\infty}$ described in $\S 2 \mathrm{~B}$. By infinitesimal asymmetry and compactness of $M$ there must be $r<\infty$ such that the $r$-jet invariant mapping $i_{\omega}^{r}$ : $Y \rightarrow J_{F}^{r}$ has image in the open set $W^{r}$ of $\S 2 \mathrm{D}$.

Define $V^{r}$ as the space of orbits of $H$ on $W^{r}$, and let $V_{A}^{r}=\left(W^{r} \cap J_{A}^{r}\right) / H \subset V^{r}$. There is a mapping of principal $H$-bundles

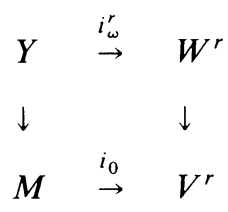

(this diagram defines $i_{0}$ ), and $i_{0}(M) \subset V_{A}^{r}$. This map $i_{0}$ clearly determines up to equivalence the bundle $Y \rightarrow M$ and the connection $\omega$.

The Pfaffian system $\psi_{1}, \ldots, \psi_{K}$ on $W^{r}$ which was described in $\S 2 \mathrm{D}$ is easily seen to push down to a Pfaffian system $\eta_{1}, \ldots, \eta_{K}$ on $V^{r}$ which has the same rank, since the $\psi_{i}$ annihilate tangents to the $H$-orbits and the system $\left(\psi_{i}\right)$ behaves apropriately under the action of $H$. Any immersion $i^{\prime}: M \rightarrow V^{r}$ which satisfies the Pfaffian system $\left(\eta_{i}\right)$ and is homotopic to $i_{0}$ clearly lifts to an $H$-equivariant immersion $f: Y \rightarrow W^{r}$ which satisfies $\left(\psi_{i}\right)$.

Remembering the immersion condition on $(\pi \circ f)$ in Lemma (2) of $\$ 2 \mathrm{D}$, we put additional structure on $V^{r}$. Since the action of $H$ preserves the fibering of $J_{F}^{r}$ over $J_{F}^{r-!}$, there is a well-defined submersion $\tilde{\pi}: V^{r} \rightarrow \pi\left(W^{r}\right) / H$, and $\pi \circ f$ is an immersion if and only if $\tilde{\pi} \circ i^{\prime}$ is an immersion. Then by $\S 2 \mathrm{D}$ we have 
LeMma 1. A smooth map $i^{\prime}: M \rightarrow V^{r}$ which is homotopic to $i_{0}$ lifts to the invariant mapping of a connection on $Y$ in geometry $A$ if and only if

(1) $\left\langle\psi_{i}, i_{*}^{\prime} X\right\rangle=0$ for all $X \in T_{*} M$ and $i=1, \ldots, K$,

(2) $\tilde{\pi} \circ i^{\prime}$ is an immersion, and

(3) $i^{\prime}(M) \subset V_{A}$.

Now we will make use of the global asymmetry to choose a particular immersion $i^{\prime}$ of this type to represent each equivalence class of structures which approximates the given one. To make this choice we use a "tubular neighborhood of the immersed submanifold" to pull back $\psi_{i}$ to a system on the normal bundle. Our procedure will automatically eliminate compositions of allowable immersions with diffeomorphisms near the identity. However, new discrete global symmetries can appear in the deformed structures unless we take some care in limiting the size of the tubular neighborhood. Thus we need the following estimate:

LEMMA 2. Let $V=V^{r}$ and $M$ be given Riemannian metrics so that $i_{0}$ is an isometry. Then there is $\delta_{0}>0$ such that each $\left\{y: d(x, y) \leqslant 2 \delta_{0}\right\}$ is a disk embedded by $i_{0}$, and such that if $0<\delta<\delta_{0}$ and $i^{\prime}, i^{\prime \prime}: M \rightarrow V$ are maps with $d\left(i_{0}(x), i^{\prime}(x)\right)+$ $d\left(i_{0}(x), i^{\prime \prime}(x)\right)<\delta / 3$ for all $x \in M$, then any diffeomorphism $h: M \rightarrow M$ with $i^{\prime} \circ h$ $=i^{\prime \prime}$ must satisfy $d(x, h(x))<\delta$, all $x \in M$.

Proof (By COntradiction). Suppose $0<\delta_{1}$ with $d\left(i_{0} x, i_{0} y\right)>\frac{2}{3} d(x, y)$ whenever $d(x, y)<\delta_{1}$, and suppose also that the first condition on $\delta_{0}$ holds for all $\delta$ with $0<\delta \leqslant 5 \delta_{1}$. Choose $\delta_{2}>0$ so that for each $x \in M$, each path component of $\left\{y \in M: d\left(i_{0} x, i_{0} y\right)<\delta_{2}\right\}$ is contained in a ball of radius $\delta_{1} / 3$, and let $\delta_{3}$ satisfy $0<6 \delta_{3}<\delta_{2}$. Choose a finite set $A \subset M$ such that each point of $M$ is within a distance of $\delta_{3}$ of some point of $A$. Now for each $k=4,5, \ldots$, choose maps $i_{k}^{\prime}, i_{k}^{\prime \prime}$ and diffeomorphisms $h_{k}$ which contradict the condition in the conclusion for values $\delta_{k}$ of $\delta$ with $\delta_{k} \downarrow 0$; by taking a subsequence we may assume that the sequence $h_{k}(a)$ converge for $a \in A$.

Since $i_{k}^{\prime} \circ h_{k}=i_{k}^{\prime \prime}$ we know for each $x \in M$ that

$$
d\left(i_{0}(x), i_{0} h_{k}(x)\right) \leqslant d\left(i_{0}(x), i_{k}^{\prime \prime}(x)\right)+d\left(i_{k}^{\prime} h_{k}(x), i_{0} h_{k}(x)\right)<\frac{2}{3} \delta_{k} .
$$

Thus if $d\left(x, h_{k}(x)\right)<\delta_{1}$ for all $x \in M$, then the first condition on $\delta_{1}$ implies that $d\left(x, h_{k}(x)\right)<\delta_{k}$, contradicting the hypothesis on $h_{k}$. It follows that $h_{k}(x)$ cannot be a Cauchy sequence for all $x \in M$ : in fact, the limit would then define a covering map $h_{\infty} \neq$ id with $i_{0} h_{\infty}=i_{0}$, and $h_{\infty}$ would be one-to-one since only finitely many sheets of $i_{0}(M)$ pass through a given point of $V$. In particular, we cannot have large $k_{0}$ such that for $k, k^{\prime}>k_{0}, d\left(h_{k}(x), h_{k^{\prime}}(x)\right)<\delta_{1}$ for all $x$, for then the above estimate and the first condition on $\delta_{1}$ would imply that $d\left(h_{k}(x), h_{k^{\prime}}(x)\right)<\delta_{k}+\delta_{k^{\prime}}$, for all $x$.

For $a \in A$, where the $h_{k}$ converge, we have $d\left(h_{k}(a), h_{k^{\prime}}(a)\right)<\delta_{1} / 3$ when $3\left(\delta_{k}+\delta_{k^{\prime}}\right)<\delta_{1}$. For arbitrary $x \in M$, choose a path $x_{t}, 0 \leqslant t \leqslant 1$, with $x_{0}=a \in A$, $x_{1}=x$, and $d\left(a, x_{t}\right)<\delta_{3}$ for all $t$. We can show that the path $h_{k}\left(x_{t}\right)$ is contained in the ball of radius $\delta_{1} / 3$ about $h_{k}(a)$, so that the absurd estimate $d\left(h_{k}(x), h_{k^{\prime}}(x)\right)<\delta_{1}$ 
holds when $3\left(\delta_{k}+\delta_{k^{\prime}}\right)<\delta_{1}$. In fact, if we note that

$$
\begin{aligned}
d\left(i_{0}(a), i_{0} h_{k}\left(x_{t}\right)\right) \leqslant & d\left(i_{0}(a), i_{0}\left(x_{t}\right)\right)+d\left(i_{0}\left(x_{t}\right), i_{k}^{\prime \prime}\left(x_{t}\right)\right) \\
& +d\left(i_{k}^{\prime} h_{k}\left(x_{t}\right), i_{0} h_{k}\left(x_{t}\right)\right) \\
< & \delta_{3}+\delta_{k}+\delta_{k},
\end{aligned}
$$

so that $d\left(i_{0} h_{k}\left(x_{0}\right), i_{0} h_{k}\left(x_{t}\right)\right)<6 \delta_{3}<\delta_{2}$ for all $t, 0 \leqslant t \leqslant 1$, then we can use the condition on components of sets $\left\{y: d\left(i_{0}(y), i_{0}(x)\right)<\delta_{2}\right\}$. This proves the lemma.

The normal bundle of an immersion $i_{0}: M \rightarrow V$ is the vector subbundle $E$ of $i_{0}^{*}\left(T^{*} V\right)$ given by

$$
E_{x}=\left\{i_{0}^{*}(\theta): \theta \in T_{i_{0}(x)}^{*}(V),\left\langle\theta, i_{0 *}(v)\right\rangle=0 \text { for all } v \in T_{x}(M)\right\} .
$$

We write $p_{E}$ for the projection of $E$ and $\sigma_{0}$ for the zero section. Without specifying a metric on $V$, we can define a tube of $i_{0}$ as an immersion $\varphi: U \rightarrow V$ of a neighborhood of $m\left(\sigma_{0}\right) \subset E$ into $V$ such that $\varphi$ extends smoothly to $\bar{U}$ so as to be injective on each $E_{x} \cap \bar{U}$ and such that $\left\langle\theta,(\partial / \partial t) \varphi\left(i_{0}^{*}(t \theta)\right)_{t=0}\right\rangle$ is positive for all $\theta \in E$. If $d\left(i_{0} p_{E}(y), \varphi(y)\right)<\delta$ for all $y \in U$, in the metric used in Lemma 2, then we will call $\varphi$ a $\delta$-tube. As a straightforward consequence of Lemma 2 we then have

LEMMA 3. If $\varphi$ is a $\delta$-tube of $i_{0}$ for $0<10 \delta<\delta_{0}$ where $\delta_{0}$ is as in Lemma 2, and if $\sigma^{\prime}, \sigma^{\prime \prime}$ are distinct sections of $E$ with image in $U$, then there is no diffeomorphism $h$ : $M \rightarrow M$ (i.e. distinct from the identity) for which $\varphi \circ \sigma^{\prime} \circ h=\varphi \circ \sigma^{\prime \prime}$.

To complete the proof of Theorem 1, we now observe that condition (2) of Lemma 1 does not need to be reflected explicitly in the final formulation:

Lemma 4. A tube $\varphi$ of $i_{0}$ can be chosen so that $\tilde{\pi} \circ \varphi \circ \sigma$ is an immersion for each section $\sigma: M \rightarrow U$.

Proof. Construct a tube first for $\tilde{\pi} \circ i_{0}$ and use the fact that $\tilde{\pi}$ is a submersion.

For later reference we also note that there is a principal $H$-bundle $\tilde{U} \rightarrow U$ to which the tube $\varphi$ lifts in an equivariant fashion. In fact, the action of $H$ on $Y$ lifts naturally to an action on $p^{*}(E) \rightarrow Y$; thus $\left(p^{*} E\right) \rightarrow E$ is itself a principal $H$-bundle. A tube $\tilde{\varphi}$ of $i^{r}(Y)$ then can be easily constructed so that the diagram

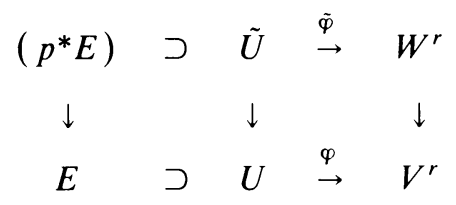

commutes and $\tilde{\varphi}$ is $H$-equivariant. A specific equivariant immersion of $Y$ is thus associated with each section $\sigma$ of $E$ with image in $U$ by the correspondence $\sigma \mapsto \tilde{\varphi} \cdot\left(p^{*} \sigma\right)$. If $\varphi \circ \sigma$ satisfies the conditions of Lemma 1, this specific immersion $\tilde{\varphi} \cdot\left(p^{*} \sigma\right)$ is the invariant mapping of a connection in geometry $A$.

\section{The universal bundle.}

A. Coordinate transformations. In the preceding section, we described a coordinate chart in a moduli space and associated to each point a particular geometric structure on $Y$. By studying the coordinate transformations, we will now fit these together to 
form a $Y$-bundle over the global moduli space (of asymmetric objects) with a structure on each fiber.

Choosing an $H$ principal bundle $Y$ over a compact manifold $M$ of the right dimension, we construct the asymmetric part $\mathfrak{N}^{\infty}(Y, A)$ of the moduli space of geometry $A$ structures on $Y \rightarrow M$. As a set, $\mathfrak{K}^{\infty}=\mathfrak{K}^{\infty}(Y, A)$ consists of the classes of infinitesimally asymmetric, globally asymmetric geometry $A$ structures on $Y \rightarrow M$ under the relation of equivalence defined in $\S 1$. $\Re^{\infty}$ is filtered by subsets $\Re^{4} \subset$ $\mathfrak{M}^{5} \subset \cdots \subset \mathfrak{K}^{\infty}$, where $\Re^{r}$ consists of the classes of those structures whose $r$-jet mappings have images in $W^{r}$.

To topologize $\mathfrak{R}^{\infty}$, we will define the basic open neighborhoods of its points in a noninvariant way and later justify our definition by showing its independence of choices. For $[\omega] \in \mathfrak{N}^{r} \subset \mathfrak{N}^{\infty}$, we choose a tube $\varphi: U \rightarrow V^{r}$ of an immersion $i_{0}$ : $M \rightarrow V^{r}$ representing the equivalence class of $A$-objects $[\omega]$; we write $\left\{i_{0}\right\}:=[\omega]$. For each $\bigodot^{\infty}$ open neighborhood $\mathscr{U}$ of the zero section in the space of sections of the normal bundle $E(\S 3)$, a basic neighborhood of $[\omega]$ in $\mathfrak{N}^{\infty}$ is given as the set of all classes $\{\varphi \circ \sigma\}$ represented by immersions $\varphi \circ \sigma: M \rightarrow V^{r}$ with $\sigma \in \mathcal{U}$ and (im $\left.\sigma\right) \subset$ $E_{A} \cap U$.

Given that this is a consistent definition of the topology, it will be appropriate to call the maps $\varphi_{*}: \sigma \mapsto\{\varphi \circ \sigma\}$ coordinate charts. The required justification amounts to showing that the "coordinate transformations" are homeomorphisms. The same must be checked for the bundle coordinate transformations, so we first describe the bundle.

There is a coordinate chart of the bundle for each of the lifted tubes $\tilde{\varphi}$ described in the last remark of $\$ 3$. We make the bundle space out of these charts by gluing, so we must specify the identifications. For each coordinate chart $\varphi_{*}: \mathcal{Q} \rightarrow \mathcal{V}$ in $\mathfrak{K}^{\infty}$ and lift $\tilde{\varphi}: \tilde{U} \rightarrow W^{r}$ of $\varphi$ as in the remark above, we obtain a map of $\mathscr{U} \times Y$ to $W^{r}$ given by

$$
(\sigma, y) \mapsto\left\{\tilde{\varphi} \circ p^{*}(\sigma)\right\}(y) .
$$

If $r^{\prime} \geqslant r$ and another chart $\varphi_{*}^{\prime}: \mathscr{U}^{\prime} \rightarrow \mathcal{V}^{\prime}$ is given with $\mathcal{V} \cap \mathcal{V}^{\prime} \neq \varnothing$, where $\varphi^{\prime}$ : $U^{\prime} \rightarrow V^{r^{\prime}}$, then let $\varphi\left(\mathcal{Q}_{0}\right)=\mathscr{V} \cap \mathcal{V}^{\prime}=\varphi^{\prime}\left(\mathcal{U}_{0}^{\prime}\right)$. The points $(\sigma, y) \in\left(\mathcal{U}_{0} \times Y\right)_{\tilde{\varphi}}$ and $\left(\sigma^{\prime}, y^{\prime}\right) \in\left(\mathscr{U}_{0}^{\prime} \times Y\right)_{\tilde{\varphi}^{\prime}}$ will then be identified if and only if:

(i) $\sigma$ and $\sigma^{\prime}$ correspond by $\varphi_{*}(\sigma)=\varphi_{*}^{\prime}\left(\sigma^{\prime}\right)$;

(ii) $\left\{\tilde{\varphi} \circ p^{*}(\sigma)\right\}(y)=p_{r, r^{\prime}}\left\{\tilde{\varphi}^{\prime} \circ p^{*}\left(\sigma^{\prime}\right)\right\}\left(y^{\prime}\right)$ where $p_{r, r^{\prime}}: J_{F}^{r^{\prime}} \rightarrow J_{F}^{r}$ is the projection.

Once we know that the maps $\left(\mathscr{U}_{0} \times Y\right)_{\tilde{\varphi}} \rightarrow\left(\mathcal{U}_{0}^{\prime} \times Y\right)_{\tilde{\varphi}^{\prime}}$ are homeomorphisms, we can define the total space $\mathcal{E}^{\infty}(Y, A)$ of our bundle as the space obtained from the disjoint union of the $(\mathscr{U} \times Y)_{\tilde{\varphi}}$ by identifying as above. The coordinate charts will then be the natural identification maps $\tilde{\varphi}_{*}: \mathscr{Q} \times Y \rightarrow \mathcal{E}^{\infty}(Y, A)$, and the group of the bundle will be $\operatorname{Diff}(Y)^{H}$. That is, to complete the proof of the following theorem we now need only check certain continuity requirements.

THEOREM 2. Let $Y \rightarrow M$ be an $H$ principal bundle over a compact manifold of the appropriate dimension. There exists a topological space $\Re^{\infty}(Y, A)$ whose points are the equivalence classes of infinitesimally and globally asymmetric geometry $A$ structures on $Y \rightarrow M$; convergence in this space means $C^{\infty}$ convergence on compact sets for some representative connection forms on $Y$. 
Over $\mathfrak{M}^{\infty}(Y, A)$ there is a bundle with fiber $Y$, group $\operatorname{Diff}(Y)^{H}$, and projection $\Pi$ : $\mathcal{E}^{\infty}(Y, A) \rightarrow \mathfrak{T}^{\infty}(Y, A)$. This bundle has the additional structure of a continuous mapping inv: $\mathcal{E}^{\infty}(Y, A) \rightarrow J_{A}^{\infty}$ such that for each class $[\omega] \in \mathfrak{N}^{\infty}(Y, A)$, the restriction (inv $\mid \Pi^{-1}\{[\omega]\}$ ) is the invariant mapping for a geometry A structure on $\Pi^{-1}\{[\omega]\}$ $\cong Y$ which represents $[\omega]$.

The structure on each fiber is of course well defined due to the asymmetry.

We have reduced the theorem to a few easy estimates which we now make explicit. By continuity of addition of sections of $E, p^{*}(E)$, etc., we can restrict our attention to continuity at the zero section for those coordinate transformations which fix the zero section. Moreover, we need only consider the case of the total space transformations, since then the result about the base will follow. Each such transformation is a composition of two types: those with $r=r^{\prime}$, and those where $r=r^{\prime}-1$ and the tube $\tilde{\varphi}^{\prime}$ is obtained from $\tilde{\varphi}$ in a trivial fashion (or vice versa).

When $r=r^{\prime}$, the map $h=\tilde{\varphi}^{\prime-1} \circ \tilde{\varphi}$ is defined in a neighborhood (say $U$ ) of the zero section of $E$ as an $H$-equivariant but nonlinear diffeomorphism which fixes the zero section. Write $\|\cdot\|_{r, K}$ for the pointwise norm on the partials of order $\leqslant r$ on compact $K$, where the differentiations are with respect to the vectorfields $X_{1}, \ldots, X_{N}$ on $Y$ given by the structure for the zero section. We then need only the obvious estimate that $\|h \circ \sigma\|_{r, K} \leqslant C\|\sigma\|_{r, K}$ for small $\sigma$.

Now let $r=r^{\prime}-1$ and define $\tilde{\varphi}^{\prime}$ from $\tilde{\varphi}$ by using the product structure on $J_{F}^{r^{\prime}} \cong J_{F}^{r} \times \mathbf{R}^{t}$, first to lift $\tilde{\varphi}$ to $J_{F}^{r^{\prime}}$ so that $\tilde{\varphi}\left(p^{-1}\{y\} \cap U\right)$ is horizontal for $y \in Y, p$ : $E \rightarrow Y$, and then to extend this lift vertically by means of an isomorphism $E^{\prime} \cong E \times$ $\mathbf{R}^{t}$. In one direction the coordinate transformation is then norm-decreasing. In the reverse direction, a section $\sigma$ of $E$ is mapped to a section $\sigma \times f$ of $E \times \mathbf{R}^{t}$. The function $f$ is defined by differentiating the function $\left(\tilde{\varphi} \circ \sigma-\tilde{\varphi} \circ \sigma_{0}\right)$ with respect to the vectorfields $X_{k}(\sigma)$ on $Y$ given by the structure associated with $\tilde{\varphi} \circ \sigma ; \sigma_{0}$ is here the zero section. As the $X_{k}(\sigma)$ are bounded relative to the $X_{j}=X_{j}\left(\sigma_{0}\right)$, there is again no difficulty with $e^{\infty}$ continuity, and the theorem is proved.

Note that the connection forms on the fibers combine to form a continuous function from a $T_{*} Y$ bundle over $\mathfrak{R}^{\infty}$ to $\mathbf{R}^{N}$.

B. Universality. We now describe the characteristic property of the object we have just constructed in Theorem 2.

Definition. A deformation of infinitesimally and globally asymmetric geometry $A$ structures on an $H$ principal bundle $Y \rightarrow M$ is a bundle with fiber $Y$ and with an infinitesimally and globally asymmetric structure on each fiber, such that each of the invariants of these structures defines a continuous function on the total space.

THEOREM 3. For any deformation $D \rightarrow X$ as above, there is a unique map of bundles

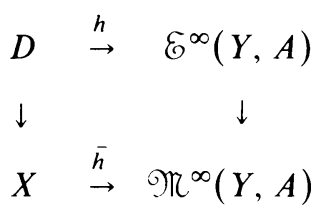

for which $h$ pulls back the canonical connection on the fibers of $\mathcal{E}^{\infty}$ to the given one on the fibers of $D$. 
Proof. It is clear that the map $\bar{h}$ is uniquely defined as a function, and that there exists a unique function $h$ so that the diagram commutes and (inv) $\circ h$ restricts to the invariant map on each fiber.

Given $r<\infty$, let $D^{r} \rightarrow X^{r}$ be the restriction to the largest $X^{r} \subset X$ mapping to $\Re^{r}$, and let $j^{r}: D^{r} \rightarrow W^{r}$ be the map $z \mapsto[r$-jet of $\operatorname{inv}(h(x))]$. We know, for every $r<\infty$ and $z \in D^{r}$ and every open neighborhood $Q^{r}$ of $j^{r}(z)$ in $W^{r}$, that $z$ has an open neighborhood in $D^{r}$ which maps into $Q^{r}$ by $j^{r}$.

Continuity of $h$ will follow quickly from continuity of $\bar{h}$ since the topology on a bundle chart is the product topology, and since the charts in $\mathcal{E}^{\infty}$ are given by tubes in $W^{r}$. Continuity of $\bar{h}$ can be expressed in terms of $h$ with fiberwise-saturated open subsets of $\mathcal{E}^{\infty}$ and $D$.

If $Y_{x}$ is the fiber over $x \in X$, let $\tilde{\varphi}_{*}: \mathcal{Q} \rightarrow \mathcal{E}^{\infty}$ be a chart centered along the fiber $h\left(Y_{x}\right)$, where $\varphi$ is a tube in $W^{r}$. The basic open fiberwise saturated neighborhoods of $h\left(Y_{x}\right)$ are given, using the equations of Lemma 1 of $\S 3$, by $e^{s}$ neighborhoods of the zero section of the source bundle of $\varphi$, for varying $s$. The solutions of these equations satisfying a $e^{s}$ estimate are, however, the same as the solutions satisfying the condition that their natural lifts to $W^{r+s}$ satisfy a $\bigodot^{0}$ estimate. The fiberwise saturated neighborhoods of $h\left(Y_{x}\right)$ are thus determined by $H$-invariant open neighborhoods $\tilde{Q}^{r}$ of $j^{r}\left(Y_{x}\right)$ where $r \geqslant r_{0}$ is allowed to vary.

Suppose such an open set $\tilde{Q}^{r}$ is given, and let $K \subset Y_{x}$ be a compact subset which meets each $H$-orbit in $Y_{*}$. Each point of $K$ has an open neighborhood mapping into $\tilde{Q}^{r}$; we may take a finite subcover $V_{1}, \ldots, V_{q}$ of $K$. The intersection of the images of the $V_{j}$ in $X$ is an open neighborhood of $x$ which is mapped by $\bar{h}$ into the appropriate open neighborhood of $\bar{h}(x)$. That is, if $p: D \rightarrow X$ is the projection, then $h$ maps $p^{-1}\left(\cap{ }_{j} p\left(V_{j}\right)\right)$ into $\tilde{Q}^{r}$.

Department of Mathematics, Rice University, Houston, TeXas 77001 